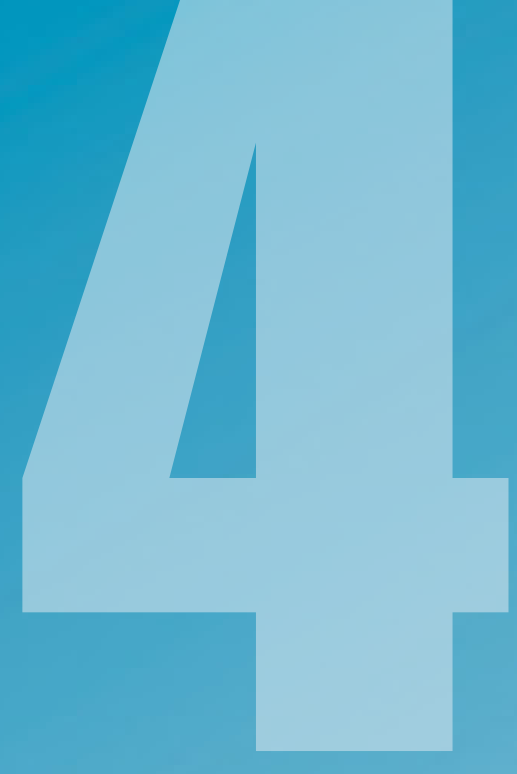

ÁMBITOS

REVISTA

INTERNACIONAL

DE COMUNICACIÓN

$N^{\circ} 46$

EDICIÓN OTOÑO

2019

ISSN: 1139-1979

E-ISSN: 1988-5733

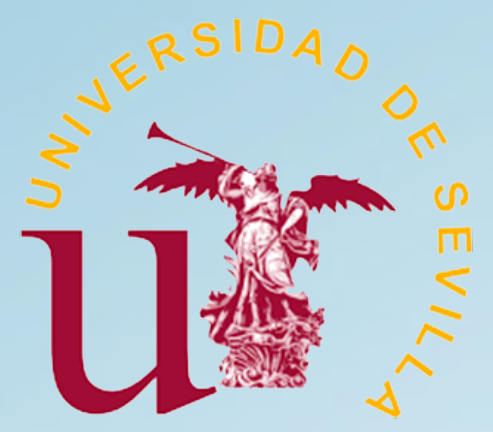




\section{ÍNDICE}

MONOGRAFICOS MONOGRAPHS

Presentación Monográfico. Comunicación emergente. Experiencias para el cambio social

Dra. Nereida López Vidales

Los formatos de televisión más consumidos por los jóvenes: telerrealidad y empoderamiento de la audiencia

Most consumed televisión formats by young people: real TV and the empowerment of the audience Nereida López Vidales, Leire Gómez Rubio, Elena Medina de la Viña

Nuevas herramientas, viejas costumbres El Contenido Generado por los Usuarios sobre el cambio climático en YouTube

New tools, old habits User Generated Content about climate change on YouTube

David Vicente Torrico

Tratamiento periodístico de personas LGTBIQ+ refugiadas: estudio de caso sobre Pride Barcelona 2018

Journalistic treatment of LGTBIQ+ refugees: case study on Pride Barcelona 2018

Hadriel Theodoro, Amparo Huertas Bailén

Tiempo Muerto, estudio de caso de un proyecto transmediático para la consecución de competencias universitarias

Tiempo Muerto, case study of a transmediatic project for the achievement of university competences Jose L. Carreño Villada, Miguel Ángel Díaz Monsalvo

Los universitarios millennials: uso de redes sociales y relación con las marcas

University Millennials: use of social media and engage with brands

Pedro Pablo Marín Dueñas, Esther Simancas González

Ali Ferzat: De la caricatura comunicativa en papel a la caricatura activista en los medios digitales

Ali Ferzat: From political cartoons in papers to political cartoons in digital media

Salud Adelaida Flores Borjabad

Direito à informação e literacia midiática: Reflexões sobre a questão do acesso

Right to information and media literacy: Reflections on the question of acce

Christiane Delmondes Versuti 
Eficacia de los influencers como recurso publicitario en la estrategia de los anunciantes locales Efficacy of influencers as an advertising resource in the strategy of local advertisers

Isabel Iniesta-Alemán

As redes sociais como ferramenta de marketing em instituições de ensino superior no Brasil Social networks as a marketing tool in higher education institutions in Brazil

Alcino Ricoy JR, Rogério Eduardo Rodrigues Bazi

Estudio sobre la conceptualización y el tratamiento informativo de la violencia de género en la prensa digital cubana

Study about the conceptualization and information treatment of gender violence in the Cuban digital press

Regla Ismaray Cabreja Piedra, Karina Escalona Peña

\section{ARTÍCULOS ARTICLES}

Uso de Twitter durante los debates electorales televisados en los comicios andaluces de 2018 The use of Twitter during the televised electoral debates in the 2018 Andalusian elections Julia Fontenla Pedreira, Erika Conde Vázquez, Carmen Máiz Bar

Quem averigua as notícias, os algoritmos ou jornalistas? A lógica crítica de C. S.

Peirce como processo de identificação de uma Fake News

Who checks the news, algorithms or journalists? The critical logic of C. S. Peirce as a

process for identifying a Fake News

Adelino de Castro Oliveira Simões Gala, Vania Baldi, Universidad de Aveiro

\section{RESEÑAS REVIEWS}

Nuevo ecosistema comunicativo digital: El consumidor

Juan Carlos Figuereo Benítez

Corpus toponímic de Beniarrés

Bianca Sánchez-Gutiérrez

The Future Computed. La inteligencia artificial y su papel en la sociedad y Pulsa actualizar. La aventura de redescubrir el alma de Microsoft y concebir un futuro mejor para todos 


\title{
Tiempo Muerto, estudio de caso de un proyecto transmediático para la consecución de competencias universitarias
}

\author{
Tiempo Muerto, case study of a transmediatic project for the \\ achievement of university competences
}

Jose L. Carreño Villada, Universidad Europea Miguel de Cervantes, 47012

email_jlcarreno@uemc.es | Orcid: https://orcid.org/0000-0003-3403-7816

Miguel Ángel Díaz Monsalvo, Universidad Europea Miguel de Cervantes, 47012 email_madiaz@uemc.es | Orcid: https://orcid.org/0000-0003-2896-551X

DOI: http://dx.doi.org/10.12795/Ambitos.2019.i46.05

\begin{abstract}
Resumen
El modelo universitario actual establece un estándar de evaluación basado en la consecución de competencias como modo de representación de la adquisición de conocimientos. Por su parte, en el ámbito de la comunicación audiovisual, las narrativas transmedia han surgido como un modelo de comunicación que hace uso de diferentes lenguajes y códigos dentro del engranaje audiovisual digital. La relación transversal de los dos ámbitos hace posible establecer una sinergia en la que la realización de un tipo de proyecto concreto de como resultado la consecución de dichas competencias preparando de forma positiva al alumno dentro de la Sociedad del Conocimiento.
\end{abstract}

En esta experiencia docente se pone de manifiesto el proyecto transmedia Tiempo Muerto Webserie, realizado por un grupo de alumnos de $4^{\circ}$ de Grado en Comunicación Audiovisual. Se abordan aspectos como la construcción de un relato transmedia, sus 
pistas de migración, los microrrelatos paralelos o la participación del usuario. Además, se valora de forma cuantitativa tanto el índice de audiencia como el grado de satisfacción del alumnado en cuanto a la consecución de competencias. La metodología cualitativa permite profundizar en las dinámicas de trabajo grupal y aspectos vinculados con el futuro laboral del estudiante tras la realización del proyecto, que supone un punto de encuentro de los conocimientos aprendidos durante los cuatro años de su etapa universitaria. Los resultados obtenidos a lo largo de seis años académicos, culminados con el presente estudio de caso, ponen de relieve tanto las herramientas transmedia como la inclusión de estrategias educativas innovadoras.

\section{Abstract}

The current university model establishes an evaluation standard based on the achievement of competences as a way of representing the acquisition of knowledge. For its part, in the field of audiovisual communication, transmedia narratives have emerged as a communication model that makes use of different languages and codes within the digital audiovisual gear. The cross-sectional relationship of the two areas makes it possible to establish a synergy in which the realization of a specific type of project results in the achievement of these competencies, preparing the student positively within the Knowledge Society.

In this classroom experience, the Transmedia Tiempo Muerto Webserie project is developed by a group of 4th grade students in Audiovisual Communication. It shows aspects such as the construction of a transmedia story, its migration tracks, parallel micro-stories or user participation. In addition, the audience level or satisfaction degree of the students towards the achievement of competences is assessed quantitatively. The qualitative methodology allows deepening the dynamics of group work and aspects related to the student's future work when they have finished the project, which is a meeting point for the knowledge learned during the four years of his university stage. The results collected during six academic years, culminated with the present case study, highlight both the transmedia tools and the inclusion of innovative educational strategies.

Palabras clave: Transmedia, competencias, webserie, comunicación audiovisual, ABP.

Keywords: Transmedia, competitions, webseries, audiovisual communication, story, $A B P$.

\section{INTRODUCCIÓN}

Las narrativas transmedia se han convertido en una forma cada vez más habitual de enfrentarnos a producciones de ficción en el ámbito televisivo y digital. Las historias traspasan las fronteras de una única plataforma y se desarrollan y expanden a través de numerosas ramificaciones, donde solo los conocimientos de todas éstas nos ayudan 
a entender el producto audiovisual de forma completa. Los macrouniversos narrativos comprenden el texto raíz, o narrativa principal, y se expanden hasta las historias personales pasadas o futuras de los personajes que las protagonizan. Podemos hablar, entonces, de transversalidad del contenido narrativo de una historia.

En la educación nos encontramos con el mismo reto. En alumno debe comprender el macrouniverso que compete a su proceso de enseñanza-aprendizaje desde el momento en el que accede al sistema universitario. Además, todo aquello que va aprendiendo a lo largo de sus estudios debe ponerse en práctica a medida que va avanzando por este camino para finalmente poder entender la Comunicación Audiovisual de una forma global.

La enseñanza y puesta en práctica de las narrativas transmedia en el aula son un potente recurso, hoy, no sólo para la adquisición de competencias, sino también para hacer uso de todo lo que el alumnado ha visto en la universidad; por eso, se ha propuesto la creación de una narración transmedia en el aula como experiencia educativa.

\subsection{Objetivos}

A la hora de dar forma a esta experiencia educativa se ha partido de un objetivo general primigenio que emana de la propia esencia de lo transmedia: la adquisición de competencias de forma integral en el marco del sistema universitario vigente, basado en la transversalidad de conocimientos teórico-prácticos, según se plasma en el Espacio Europeo de Educación Superior.

Desde esta base se postulan otros objetivos, como constatar la importancia de nuevas fórmulas de enseñanza-aprendizaje en el sistema de educación superior (trabajo autónomo del alumno, generación de grupos de trabajo compuestos por perfiles diferentes, y aplicación del Aprendizaje Basado en Proyectos (ABP) en un caso concreto), o imbuir a los alumnos de las herramientas necesarias para ejecutar una construcción narrativa basada en los postulados transmedia.

\subsection{Hipótesis}

Como punto de partida, se establece que la puesta en práctica de narrativas transmedia refuerzan el aprendizaje integral de los alumnos del Grado en Comunicación Audiovisual. Pero, además, existen otras ideas relevantes que subyacen de dicha afirmación, entre ellas y como más relevante, que es posible crear una narrativa transmedia robusta con independencia presupuestaria y que las prácticas basadas en casos reales motivan al alumno a enfrentarse al mundo laboral el día de mañana, trascendiendo el ámbito universitario. 


\subsection{Metodología}

Para cimentar la experiencia educativa se ha construido un armazón basado en un marco teórico dual, que abarca tanto las bases de la narrativa transmedia como la configuración del proceso de enseñanza-aprendizaje en el ámbito universitario.

La metodología es mixta. En una primera fase, predomina una vertiente cualitativa, basada en el análisis de contenido de los planes de estudio de las universidades españolas presentes en los primeros puestos del U-Ranking 2018 (Pérez y Aldás, 2018) como son la Universitat Popeu Fabra, Universidad Carlos III, Universidad Autónoma de Madrid y Universitat Autónoma de Barcelona, por ser las primeras del mismo, en busca de asignaturas que estén vinculadas de forma directa a esta forma de construcción mediática. Dado que no se explicita ninguna materia específica, se han extraído las competencias plasmadas en las guías docentes de materias en las que se trabaja el ámbito transmedia, cotejándolas con las de las asignaturas propias que han servido para evaluar el trabajo grupal del presente estudio de caso.

Se ha revisado, asimismo, la literatura científica acerca del Aprendizaje Basado en Proyectos, implementando sus premisas al análisis competencial previo. Con ello, se ha procedido a generar la experiencia transmedia, basada en las explicaciones teóricas sobre narrativas transmedia, la construcción de un macrouniverso ficcional, la creación de pistas de migración, el relato inmersivo y la potenciación de la interacción del usuario.

La vertiente cuantitativa ha llegado en el tramo final de la investigación. Se han tomado como referencia a los 52 alumnos que cursaron la materia en la que se enmarca el proyecto desde el curso 2013-2014 hasta el curso 2017-2018. Estos alumnos han funcionado como baremo evaluativo de la experiencia, a través de una encuesta con 23 preguntas para ser respondidas mediante escala Likert (Morales Vallejo, 2000). El producto audiovisual base de este estudio de caso ha sido sometido a un rastreo de las visualizaciones e interacciones en redes sociales para que el alumno tome consciencia de un escenario real. Los datos se han vinculado con un focus group en el que han participado sus 7 creadores, y que se ha orientado a relacionar las competencias con el proceso de creación. Los alumnos han calificado y reflexionado, no sólo acerca de la propia actividad, sino también sobre la labor del profesorado responsable y la pertinencia del proyecto de cara a su futuro profesional.

\section{LAS NARRATIVAS TRANSMEDIA EN EL ÁMBITO UNIVERSITARIO}

El uso de las narrativas transmedia se está convirtiendo en un modelo televisivo por el que apuestan las televisiones públicas y privadas, caso de las series El Ministerio del Tiempo (TVE) o La Casa de Papel (Antena 3 / Netflix). Por lo tanto, es necesario que los estudiantes vinculados al mundo de la comunicación audiovisual y el periodismo deban conocer este nuevo tipo de paradigma comunicativo en sus vertientes.

Ámbitos. Revista Internacional de Comunicación | ISSN: 1139-1979 | E-ISSN: 1988-5733, №. 46. (2019) 
La narración transmediática propone macrouniversos ficcionales que se relacionan entre ellos, donde el conocimiento de sus diferentes piezas hace que el usuario tenga un conocimiento global del producto. Tal y como afirma Mittle (2006), este tipo de formas narrativas quieren sorprender al espectador/usuario en forma y en contenido además de resultar más atractivas potenciando seguidores más fieles.

Las audiencias tienden a abrazar programas complejos en términos mucho más apasionados y comprometidos que la mayoría de las televisiones convencionales, utilizando estos programas como base para una cultura de fans sólida y una respuesta activa a la industria de la televisión (especialmente cuando sus programas están en peligro de cancelación). (Mittell, 2006, p.32)

Pero ¿saben los futuros profesionales de este tipo de prácticas? ¿Realmente se les está preparando en una formación integral y competitiva? La emisión del informe U-Ranking 2018 (Pérez y Aldás, 2018) muestra la posición que ocupan las diferentes universidades españolas, y, si además nos centramos en aquellas que ofrecen el Grado en Comunicación Audiovisual, los puestos quedan repartidos de la siguiente manera: Universitat Popeu Fabra, Universidad Carlos III, Universidad Autónoma de Madrid y Universitat Autónoma de Barcelona.

De estas cuatro universidades, solo en una de ellas, la primera del ranking, se le concede una importancia independiente al estudio de las narraciones transmedia; en este caso, nos referimos a la participación en un proyecto europeo que ha dado por resultado la creación del libro Transmedia literacy in the new media ecology: white paper (Scolari, 2018). Si los modelos televisivos están potenciando cambios, cabe plantearse la necesidad de sumarse a estos criterios evolutivos y asumir riesgos y cambios en el modelo educativo.

Al igual que en la televisión, en el ámbito pedagógico el aprendizaje cada vez es más cooperativo, requiriendo del uso y manejo de las TICs para la consecución de objetivos globales. De igual modo, se vuelve necesario un cambio en el paradigma evaluativo, para que de verdad se pueda cuantificar las competencias adquiridas por el alumno. La educación en etapas universitarias se considera transversal, el conocimiento de las diferentes asignaturas debe llevar al alumno a la comprensión integral de su futuro profesional, de ahí que haya una relación directa entre las diferentes asignaturas y la superación de éstas para que el alumno se pueda enfrentar de una forma más enriquecedora a sus futuros retos. El aprendizaje significativo potencia la formación integral del alumnado; por eso, es preciso que el conocimiento escalonado forme un mejor profesional de cara a su futuro laboral.

Los cambios vividos en la universidad en los últimos años, como la aplicación del conocido como plan Bolonia, han propiciado la aparición no tanto de nuevas formas pedagógicas, como de una nueva terminología a la hora de enfrentarse a los innovadores 
modelos educativos. Palabras como Aula Invertida, ABP o herramientas como Kahoot, o aplicaciones para la creación de rúbricas, RubiStar, se han convertido en modelos que muchos docentes universitarios siguen. Técnicas relegadas a modelos de educación básica, como la gamificación y ludificación dentro del aula, están a la orden del día en las enseñanzas universitarias como reseñan investigaciones tales como las de Cortizo, Carrero, Monsave et al (2011), Alexander Oliva (2016), Labrador y Villegas (2016) o Corchuelo Rodríguez (2018).

[...] la conversión de una actividad formativa académica en una experiencia lúdica atrayente y retadora no solo ha potenciado la motivación e implicación de los estudiantes para acometer la "misión" presentada de forma efectiva, sino que ha contribuido al incremento de numerosas competencias. (Villalustre y del Moral, 2016, p.29)

En medio de esta amalgama terminológica, los docentes asumen el reto de educar a sus alumnos para convertirse en futuros profesionales, de ahí que se pueda recalcar que en los modelos universitarios de medios de comunicación las narrativas transmedia aún no tengan su hueco pertinente. La producción audiovisual está dejando entrever lo enriquecedor de poseer productos donde el usuario tenga protagonismo, tal y como pasa con las narrativas transmedia. Además, la creación de este tipo de contenidos potencia el trabajo en equipo y la necesidad de diferentes perfiles profesionales para su desarrollo.

La revista científica Comunicar es la única publicación del ámbito de la comunicación con una valoración Q1 en JCR, de ahí que se haya tomado como referencia, observando que, en un gran número de artículos revisados, entre los más leídos no se ofrece una metodología concreta para hacer un uso pedagógico de narrativas transmedia, al igual que no se centra en el desarrollo de competencias múltiples que pueden tener este tipo de proyectos audiovisuales. Obviamente, debido a la temática de la revista sí se han hallado investigaciones del campo educativo en relación con los entornos digitales, fuente cercana a la narratología transmedia, como son los de Gutiérrez y Tyner (2012), Pérez-Mateo, Romero y Romeu (2014), Fernández Cruz y Fernández Díaz (2016), Pérez-Rodríguez y Delgado (2012), Marcelo, Yot y Mayo-Ruiz (2015), Romero y FanjulPeyró (2010), Rincón (2011), Cebrián Herreros (2009) o Ferrés (2012).

El aprendizaje basado en proyectos (ABP) y la ejecución de tareas reales y simuladas serán el garante de que las técnicas de innovación educativa están presentes en este tipo de dinámicas grupales. Herramientas que, por norma general, están más estudiadas en el ámbito de la educación primaria y secundaria. Estas herramientas también tienen cabida en el ámbito universitario y ponen de manifiesto la necesidad de cambiar algunas tendencias llevadas a cabo en el aula en torno a las lecciones magistrales, haciendo más participe al alumnado de su propio aprendizaje. 


\subsection{La educación dentro del marco universitario}

Desde las perspectivas constructivistas, en concreto desde la de Lev Vygotsky (Chaves, 2001), se atiende a la construcción de funciones superiores del pensamiento en relación con las interacciones culturales. Las interacciones en el aula se desarrollan a través de modelos sociales, y si se tienen en cuenta las particularidades de grados como Comunicación Audiovisual (donde el alumnado debe estar en contacto continuo con la parte cultural del mundo), se puede destacar que los alumnos, a través de sus propias búsquedas de información y autoaprendizaje, asisten a las aulas para confirmar sus conocimientos y guiar, en cierta manera, su aprendizaje.

El alumnado, con todos los medios técnicos y avances tecnológicos a su alcance, dista mucho del antiguo discípulo que escuchaba lecciones magistrales. Algunas teorías alternativas de estos nuevos aprendizajes hablan incluso de caos en el aprendizaje (Siemens, 2004), donde las aulas han evolucionado desde ese caos con el que llegan las nuevas tecnologías hasta formas auto organizadas. "Este proceso de autoorganización también se interpreta a menudo como la evolución del orden desde un comienzo desordenado" (Rocha, 1998, p.4). Las aulas han tenido que reorganizarse atendiendo a los cambios sociales y tecnológicos, han debido aferrase a las TICs para evitar el distanciamiento con su propio alumnado evitando que entre unos y otros exista una brecha no solo generacional sino también tecnológica. Autores como Cabero (1998); Duarte (1998) o González Soto (1998), han sido precursores al hablar sobre algunos matices sobre las Tecnologías de la Información y la Comunicación, asumiendo que están formadas por una pluralidad de medios que giran en torno a la comunicación, ya sea informativa o de cualquier otra índole. Características como inmaterialidad, interconexión, instantaneidad e interactividad, ligadas con las narrativas transmedia, tal y como afirman Scolari (2013) o Jenkins (2009). Esto hace posible poder crear un nexo de unión entre la implantación de las TICs con sus diferentes características y la creación de nuevos relatos en el entorno audiovisual digital.

El marco educativo universitario actual gira en derredor de la adquisición de competencias, entendiéndolas como habilidades y destrezas que el alumno debe adquirir a través del trabajo durante su etapa universitaria. Pero si se asume lo marcado por Siemens (2004) sobre la autoorganización del alumno desde un caos donde recibe información desde muchas vías diferentes, hay que entender que éstos deben llegar hasta la autogestión de su propio conocimiento:

Estudiante que deberá de estar preparado, por una parte, para el autoaprendizaje mediante la toma de decisiones, y por otra para la elección de medios y rutas de aprendizaje, y la búsqueda significativa de conocimientos. Sin olvidar su actitud positiva hacia el aprendizaje colaborativo y el intercambio de la información. (Cabero, 1998, p.145) 
El aprendizaje colaborativo, como apunta Cabero, se convierte en una punta de lanza en estas nuevas formas de aprendizaje, y más si tenemos en cuenta las particularidades de las carreras de Comunicación Audiovisual, donde el trabajo en equipo es necesario e influye directamente sobre las capacidades del alumno para poder desarrollar tareas profesionales en el futuro. A esta premisa se suma la aparición de las TICs, y aunque los apuntes de Cabero siguen estando vigentes, otros estudios hacen referencia a esta nueva situación:

En el pasar de los años no se puede decir que el aprendizaje colaborativo se ha redefinido, más bien se ha adaptado a las nuevas herramientas TIC que han surgido, como son la mensajería instantánea, los foros, las wikis, los blogs, los microblogs, las redes sociales, la marcación social, etc. Sobre cada una de estas, o su combinación, se hallaron estudios que se enfocan en demostrar la efectividad del aprendizaje colaborativo a través de ellas. (Avello y Duart, 2016, p.273)

Enraizado con este aprendizaje colaborativo están otras nuevas formas educativas como es el caso del Aprendizaje Basado en Proyecto (ABP). Son modelos de aprendizajes transversales donde se propone a los grupos un proyecto concreto a desarrollar y los pasos que deben seguir a fin de guiarles no solo hasta la consecución de un producto, sino al enfrentamiento a retos y problemas que deben ser resueltos teniendo en cuenta experiencias anteriores y las indicaciones que se han marcado en el aula antes de enfrentares a ese proyecto.

Ya hace veinte años, autores como Blank (1997) o Harwell (1997) relataban experiencias centradas en estas dinámicas dentro del aula. Hace una década, la bibliografía se actualizaba con escritos como los de Maldonado (2008) o Martí (2010), y en los últimos tiempos encontramos referencias como las de Morales (2018), Medina y Tapia (2017) o Fidalgo, Sein-Echaluce y García (2017), que entienden que este modelo ayuda a los alumnos a participar de forma activa en su aprendizaje.

El APB debe ser una de las herramientas fundamentales para el desarrollo de las competencias (creatividad, resolución de problemas, habilidad de investigar, trabajar de forma colaborativa, motivación y uso de redes sociales, entre otras) que se van a potenciar en los sistemas educativos del Siglo XXI. (Ausín, Abella, Delgado et al.,2016, p.32)

Este tipo de proyectos también están basados en la interdisciplinariedad, ya que permite trabajar con la consecución de competencias a través del desarrollo del aprendizaje significativo manifestado en diferentes asignaturas.

El rol del profesor en estos casos también se ve modificado porque su imagen frente al aula está mutando. Antes era seguido a través de las lecciones magistrales. Ahora, aunque esta vertiente deba estar presente, es necesario reestructurarla para hacerla 
valiosa para el alumno (Rodríguez Sánchez, 2011). El perfil del nuevo educador está basado en competencias que deben intentarse desarrollar en los alumnos, pero también el profesorado debe trabajar otro tipo de competencias como son las intrapersonales, las sociales o las profesionales. (Galvis, 2007).

La naturaleza de la educación es cambiante, y con cada ley pedagógica se necesita la renovación de los planes de estudio y la forma de enfrentarse al aula. Nuestra responsabilidad como docentes es reflexionar sobre nuestras actuaciones, pero no solo en el aula, sino que es necesario que tengamos en cuenta la preparación, la intervención didáctica directa y las acciones posteriores (Martínez García, García Domingo y Quintanal Díaz, 2013).

El uso de las TICs, ya mencionado, juntos con el pensamiento del uso del Aprendizaje Basado en Proyecto como dinámica transversal, tiene mucho que ver con el trabajo sobre narratología transmedia. Existen propuestas que comienzan a valorar la importancia de las narrativas transmedia en el ámbito educativo y reflexionan sobre su utilidad, eficacia e importancia. Grandía Perez (2015) marca seis dimensiones para trabajar la alfabetización transmedia en el aula desde un punto de vista crítico y otro creador, y Scolari (2016) resalta que la alfabetización transmedia se ha desplazado hacia un aprendizaje informal. Añade, en esta línea, Ambrosino (2017, p.18): "Las narrativas transmedia ofrecen oportunidades de enriquecimiento pedagógico si se conciben desde una perspectiva didáctica fuertemente comprometida con la comprensión." (p.18). Un enriquecimiento que debe ser dual, es decir, entendido desde la perspectiva tanto de profesor como de alumno. Por ello, Ossorio (2014) relaciona el uso docente de narrativas transmedia con una potenciación de la participación del estudiante. Todas estas pautas conducen a la idea de que este tipo de narrativas son la fórmula adecuada para la adquisición de cierto tipo de competencias del alumnado.

Si en la docencia nos acogemos a los principios del aprendizaje significativo como marco principal para la presentación de nuevos conocimientos, en las narrativas transmedia deberemos tener, también, un marco principal al que se asirán las diferentes incursiones en nuestro parnaso comunicativo, por lo que podemos establecer un nexo entre los aprendizajes multitarea y transversales y la creación de un relato transmedia para su exposición en diferentes ámbitos de la comunicación en plena Sociedad del Conocimiento.

\subsection{Las narrativas transmedia}

La terminología de narrativa transmedia está ligada a autores como Henry Jenkins (2009), Pratten (2015), Evans (2011) en el caso anglosajón, y Guerrero (2014), Rodríguez y Tur-Viñes (2014), Costa Sánchez y Peñeiro (2012), Scolari (2009, 2016 y 2017), Guerrero-Pico (2016), De la Fuente, Gómez y Borda (2016), en el caso de las fuentes bibliográficas que podemos acceder en castellano. Son algunos de los más 
citados. Las narrativas transmedia hablan de narraciones multiplataforma donde la suma de las partes da por resultado un intrincado narrativo donde cada aportación suma a la hora de entender la totalidad de un macrouniverso ficcional. O lo que es lo mismo, en las narrativas transmedia encontramos relatos que se desarrollan a través de diferentes plataformas, de ahí que Costa y Peñeiro (2012) afirmen lo siguiente: "Existencia de múltiples plataformas, cada una de dichas plataformas supone un punto de entrada en la historia." (p.122). Scolari y Establés (2017) definen esta forma de narración afirmando que:

Las narrativas transmedia son una particular forma del relato que se expande a través de diferentes medios y plataformas de comunicación y van mucho más allá de la simple "adaptación" de un medio a otro: la historia que cuenta el videojuego no es la misma que aparece en el cine o en la novela. (p. 1016)

Scolari (2014), en su análisis sobre la situación de las narrativas transmediáticas en España, nos habla de cómo los medios se están convirtiendo en transmedia aunque necesita una renovación tanto de los productos como de los profesionales. De ahí que resulte de especial interés manifestar estas fórmulas narrativas dentro del aula.

Pero si hay algo que está ligado a este tipo de nuevas formas creativas, donde importa la transversalidad de soportes y plataformas, es los usuarios. Los antiguos observadores pasivos pasaron hace tiempo a convertirse en actantes de la comunicación (Gubern, 2000), los nuevos medios y la interconexión entre participantes ha generado que ese público pase a llamarse usuario y que, además, tenga verdadera importancia en el proceso comunicativo. Una narrativa transmedia puede ser pensada de antemano, entendiéndose esto como nativa, pero puede irse construyendo a medida que los usuarios se apropian de ella siendo los encargados de expandirla y de generar discursos que otros usuarios quieran escuchar.

No todas las narrativas transmedia llegan al mismo lugar, algunas apenas cuentan partida presupuestaria y, por lo tanto, sus expansiones, es decir, aquellos relatos que derivan del relato principal, se hacen complicadas si no cuentan con el beneplácito de los usuarios. Hay otras, en cambio, que a través de mundos bien construidos han conseguido no solo llegar a sus usuarios sino ofrecer una experiencia enriquecedora que, además, invite a hacer partícipe a otros. Es justo en este momento donde se hace necesario atender al paradigma comunicativo de las redes sociales, un punto de encuentro donde el usuario se puede dirigir a un producto o a un actor o incluso, a otros usuarios para ser guiado en el camino narrativo. Es necesario destacar que es justo esta conectividad entre participantes lo que enriquecerá la narración. "Tanto en las narrativas transmedia como el conectivismo proponen herramientas de edición, participación, interactividad y distribución de contenidos por las redes sociales y ambientes wiki" 
(Renó, 2012, p. 206). Por lo tanto, ya tenemos algunos elementos clave para generar una narrativa transmedia como que:

- sea un relato multiplataforma

- invite al espectador a formar parte del mundo que se le ofrece

- tenga pertenencia al ámbito de las redes sociales

Liuzzi (2014) retoma la importancia de los prosumidores, esos usuarios que antes eran solo observadores, pero que han decidido saltar hacia una parte más creadora; y, además arroja algo de luz sobre las audiencias en el entorno digital. Por este motivo, recoge los datos de un estudio donde los usuarios coincidieron en destacar que eran necesarias narrativas que propiciaran:

- Inmersión: Como posibilidad de entrar de maneras mucho más profundas a la historia, aprender más sobre ella y tener una experiencia diferente.

- Interactividad: Tener la capacidad de cambiar o influenciar los elementos de la historia y poder interactuar con otros usuarios involucrados en el mundo narrativo.

- Integración: Consumir una historia que se extienda y cruce plataformas. ¿Puede existir una unión entre las interfaces y el mundo real?

- Impacto: La narrativa inspira al usuario a realizar acciones en el mundo real. (Liuzzi, 2014, p. 72)

Unas características que, si observamos, tienen mucha relación con lo citado anteriormente sobre las características de las TICs, que modifican la forma de construir los mensajes y la manera en que se deben consumir, publicitar y, por supuesto, crear los relatos.

En el siguiente apartado se desgranará cómo realizar una dinámica transmedia en el aula a través del estudio de caso de una de las webseries trabajada en el aula durante el curso 2017-2018 de los alumnos de $4^{\circ}$ de Grado en Comunicación Audiovisual, y sus consideraciones científicas y académicas. El grupo contó con una dotación técnica como para llevar a cabo una producción audiovisual de bajo presupuesto, y debía explorar las posibilidades de las TICs tanto para conseguir seguidores de sus productos audiovisuales como para crear diferentes modelos narrativos interrelacionados entre sí. A medida que el profesorado se ha ido formando en materia transmedia, el alumnado ha ido desarrollando diferentes proyectos, y por eso, finalmente, se ha centrado esta investigación en la producción de la serie realizada entre 2017 y 2018 como estudio de caso, por ser una de las experiencias más completas con las que se ha podido contar. 


\section{EXPERIENCIA EDUCATIVA: TIEMPO MUERTO WEBSERIE}

\subsection{Análisis metodológico de competencias a desarrollar}

Este proyecto se ha llevado a cabo a través de la asignatura de "Producción y Gestión de Nuevos Formatos Audiovisuales", dado que las competencias fijadas en su guía docente eran las que más encajaban con los postulados transmedia y donde mejor se insertaban las fórmulas de aprendizaje desarrolladas. En un primer momento, se han buscado asignaturas con la etiqueta 'transmedia' en las universidades que están en cabeza del U-Ranking 2018 (Popeu Fabra, Carlos III, Autónoma de Madrid y Autónoma de Barcelona). Al no existir, se han buscado las materias en cuyo plan de actuación figurasen áreas vinculadas a este tipo de narrativa, y se han cotejado las competencias que figuran en sus guías docentes respecto a nuestra asignatura donde se enmarca este trabajo, dando como resultado el siguiente marco competencial:

a) Generales: desde la capacidad de análisis y juicio crítico, pasando por el trabajo en equipo, liderazgo, resolución de problemas, desarrollo de pensamiento creativo, adaptación al entorno y nuevas situaciones, comunicación de imágenes, ideas o símbolos, y aplicación de los conocimientos a la práctica. Estas competencias son tangenciales en todas las asignaturas, y tienen una sencilla traslación al proyecto transmedia.

b) Específicas:

01. Capacidad para conocer y aplicar las técnicas y procesos de producción, difusión, distribución o exhibición audiovisual (cine, televisión, radio, publicidad, hipermedia, videojuego, animación, infografía, música, fotografía o narración gráfica).

02. Capacidad y habilidad para planificar y gestionar los recursos humanos, técnicos y presupuestarios aplicados a los distintos medios, soportes o formatos audiovisuales.

03. Capacidad para desarrollar y ejercer las distintas funciones profesionales de la producción audiovisual.

El ámbito de las competencias es importante para poder desarrollar cualquier proyecto dentro del espacio universitario, pero tiene un valor añadido si se intenta trabajar a través de un único proyecto audiovisual que se desarrollara de forma episódica.

\subsection{Desarrollo de la experiencia, biblia audiovisual, cronología y plan de preproducción}

Una vez sentadas las bases competenciales, y asumiendo las teorías del ABP y el Aula Invertida, durante tres semanas se establecieron sesiones teóricas para explicar los 
principios de la narratología transmedia, poner a disposición del alumno artículos y ejemplos para ir pensando en la experiencia a realizar. También se dotó al alumnado de ítems necesarios para la creación de una biblia audiovisual y en concreto una biblia transmedia.

A partir de este momento, el equipo contaba con 6 semanas para elaborar dicha biblia, donde debían plasmar tramas y subtramas de la webserie, referencias audiovisuales, storylines de todos los capítulos, necesidades técnicas específicas, calendario de grabación y emisión y elaboración de los elementos transmedia incluyendo pista de migración y storylines y cronología de emisión de este formato de narración. Además, se potenció la creación de redes sociales propias para cada producto de forma que sirvieran de soporte publicitario para los productos.

Dado el cariz de la asignatura, se pidió a los alumnos que este tipo de narraciones, además de los elementos transmedia propiamente reseñados, debían disponer de algún elemento que convirtiera a la narración en algo innovador que potenciara la interacción de los usuarios.

El usuario y su compromiso, o el grado de engagement de éste, es vital (como aseguran Jenkins o Scolari), de ahí que la narración debía fortalecer este compromiso, asumiendo que en el caso de este producto debía valorarse de forma más positiva el compromiso por encima de las visualizaciones. No conviene olvidar que fue el compromiso de los internautas lo que salvó a otras series transmedia como fue el caso de El Ministerio del Tiempo (Rodríguez-Mateos y Hernández-Pérez, 2015), donde TVE no solo cuantificó los resultados televisivos, sino que valoró también la audiencia social de la serie.

Para la creación de los productos se dio la posibilidad de elegir cualquier género, y de aquí salieron como propuestas tres thrillers, una comedia y un falso documental. En una producción audiovisual profesional, lo normal es que haya un director y el resto sean operadores técnicos, pero en esta dinámica los roles de los alumnos debían ir modificándose según cada capítulo. Cada alumno fue responsable de la dirección, edición y guion de 2 capítulos, ocupando otros roles técnicos en los restantes capítulos de la producción.

Por parte del profesorado, esta práctica se convirtió, además de transmedia, en trasversal, porque contó con profesores del ámbito de la Producción Sonora, Producción Audiovisual, Guion e lluminación y Escenografía para supervisar el proyecto; en unos casos de forma extracurricular, y en otros de forma curricular. Así, el aprendizaje significativo queda patente volviendo a contar con la ayuda de docentes que ya habían trabajado con los alumnos con anterioridad. 
De forma grupal los alumnos debían trabajar en torno a dinámicas de tareas reales, por eso, hicieron castings propios para encontrar a su elenco de actores. Tras esas 6 semanas, cada grupo debía mostrar su biblia a los profesores y demostrar la viabilidad del proyecto. Así nacía la webserie "Tiempo Muerto", un relato sobre un futuro distópico donde una asociación criminal intentaba acabar con los pocos habitantes que quedaban de la raza humana.

\subsection{Producción y desarrollo}

Una vez generada la biblia, los alumnos contaron con otras 7 semanas para la grabación y edición de los capítulos. Además, debían empezar a generar expectación a través de su presentación en redes sociales, incluyendo algunos de los aspectos de los que hacían que sus creaciones fueran algo innovador, ya fuera por la implicación del usuario, uso de vídeo en $360^{\circ}$ o historias donde el usuario decide como verlas.

El resultado fue una serie con un total de 14 capítulos con narrativa transmedia nativa en la que se concede al usuario una gran importancia, hasta el punto de que debía descifrar una clave para poder visionar el capítulo final de la serie, como se explicará más adelante. El siguiente cuadro muestra las diferentes pistas de migración que llevaban del relato principal hacia otra parte del macro universo narrativo, como el pasado de dos de los protagonistas en diferentes plataformas. 
Tabla 1

Elementos transmedia de Tiempo Muerto

\begin{tabular}{|c|c|c|c|c|}
\hline $\begin{array}{c}\text { Acción transme- } \\
\text { dia }\end{array}$ & Marco narrativo & $\begin{array}{c}\text { Plataforma de } \\
\text { desarrollo }\end{array}$ & $\begin{array}{l}\text { Interacción } \\
\text { necesaria del } \\
\text { usuario }\end{array}$ & $\begin{array}{c}\text { Su relación con la } \\
\text { historia }\end{array}$ \\
\hline Relato $360^{\circ}$ & $\begin{array}{l}\text { Personaje princi- } \\
\text { pal }\end{array}$ & YouTube & $\begin{array}{c}\text { Activa. Es necesaria } \\
\text { la manipulación del } \\
\text { usuario. }\end{array}$ & $\begin{array}{l}\text { Relación directa con el } \\
\text { capítulo piloto. }\end{array}$ \\
\hline Instagram Ángela & Pasado de Ángela & Instagram & $\begin{array}{l}\text { Pasiva. Después de } \\
\text { indagar y encontrar el } \\
\text { perfil el usuario } \\
\text { puede seguir solo } \\
\text { como observador. }\end{array}$ & $\begin{array}{c}\text { En el capítulo } 2 \mathrm{~A} \text { el } \\
\text { personaje recoge unas } \\
\text { fotos de su coche que se } \\
\text { relaciona con la pasión } \\
\text { del personaje por la } \\
\text { fotografía. }\end{array}$ \\
\hline Souncloud Víctor & $\begin{array}{l}\text { Pasado y pre- } \\
\text { sente de Víctor }\end{array}$ & Souncloud & $\begin{array}{l}\text { Pasiva. Después de } \\
\text { indagar y encontrar el } \\
\text { perfil el usuario } \\
\text { puede seguir solo } \\
\text { como observador. }\end{array}$ & $\begin{array}{c}\text { El personaje en sus } \\
\text { capítulos va grabando } \\
\text { audios en una grabadora. } \\
\text { En soundcloud } \\
\text { encontramos las } \\
\text { grabaciones completas. }\end{array}$ \\
\hline Blog Drome & $\begin{array}{c}\text { Se presenta a } \\
\text { miembros de la } \\
\text { asociación que } \\
\text { está detrás de la } \\
\text { situación de la } \\
\text { humanidad. }\end{array}$ & Wordpress & $\begin{array}{l}\text { Pasiva. Después de } \\
\text { indagar y encontrar el } \\
\text { perfil el usuario } \\
\text { puede seguir solo } \\
\text { como observador. }\end{array}$ & $\begin{array}{c}\text { El nombre de la } \\
\text { asociación criminal } \\
\text { aparece varias veces a lo } \\
\text { largo de la historia. }\end{array}$ \\
\hline $\begin{array}{c}\text { Estructura argu- } \\
\text { mental }\end{array}$ & Relato principal & YouTube & $\begin{array}{l}\text { Activa. Al final del } \\
\text { capítulo piloto el } \\
\text { usuario podía decidir } \\
\text { si veía el arco } \\
\text { narrativo } 1,2 \text { o } 3 \text {. }\end{array}$ & Relato principal \\
\hline Capítulo final & Conclusión & $\begin{array}{l}\text { YouTube dife- } \\
\text { renciado }\end{array}$ & $\begin{array}{l}\text { Activa. El usuario } \\
\text { solo podía acceder a } \\
\text { este capítulo } \\
\text { descifrando una } \\
\text { clave oculta en el } \\
\text { relato. }\end{array}$ & Relato principal \\
\hline
\end{tabular}

Fuente: elaboración propia 


\subsection{Estreno y visionado}

A mediados de enero de 2018, después de diferentes promociones y tráileres, se estrenaba la producción audiovisual, "Tiempo Muerto Webserie" (https://www.youtube.com/channel/UC_nBoM0G04t6t4CHYmVmPiQ). A la ficción se le adelantó un pequeño tráiler explicando su forma de consumo a los internautas. (Figura 1).

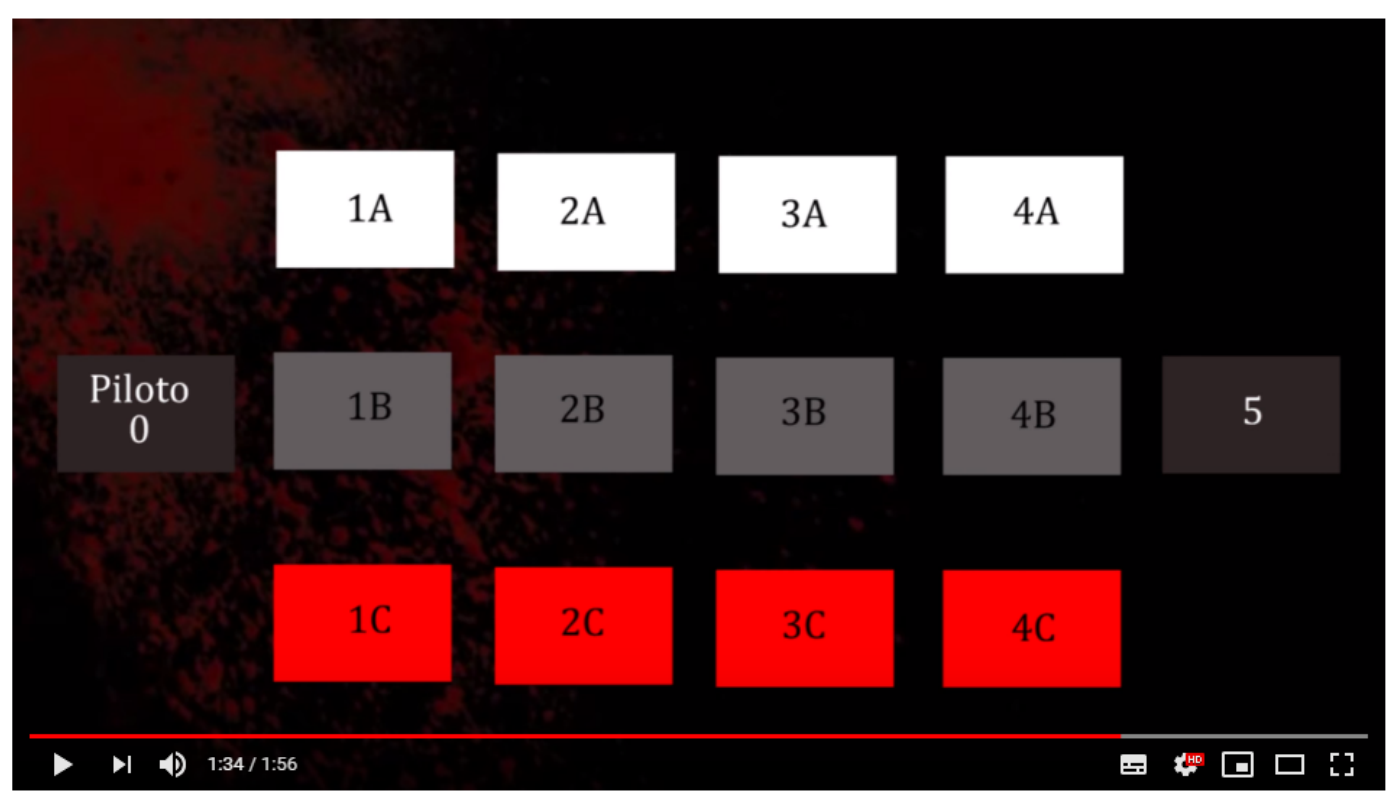

ELIGE TU CAMINO II TRÁILER 2 TIEMPO MUERTO [VÍDEO EXPLICATIVO]

Figura 1. Captura de imagen tráiler "Tiempo Muerto"

Fuente: YouTube

En la biblia presentada en el aula, el alumnado había explicado que su público objetivo se enfocaba a gente joven que consumía productos audiovisuales a través de dispositivos móviles, aunque también dudaron sobre el compromiso exploratorio de este target. Por ello, asumieron que, aunque el arranque fuese positivo, el capítulo final no tendría tan buenos resultados.

Llega, por tanto, el momento de cuantificar y contextualizar los datos del producto audiovisual resultante. La serie tuvo algo más de 8.000 reproducciones, como muestra la siguiente tabla: 


\begin{tabular}{|c|r|r|}
\hline Visualizaciones & $\begin{array}{r}\text { Tiempo de } \\
\text { visualización } \\
\text { (minutos) }\end{array}$ & $\begin{array}{r}\text { Espectadores } \\
\text { únicos }\end{array}$ \\
\hline $8.275 \quad 100,0 \%$ & $11.679 \quad 100,0 \%$ & $1.739100,0 \%$ \\
\hline
\end{tabular}

Figura 2: Análisis cuantitativo de la serie. Visualizaciones Fuente: Youtube Analitycs

En cuanto a parámetros que se habían definido en la biblia sobre el público objetivo, nos encontramos con los siguientes resultados en cuanto a edad, sexo del público y tipo de dispositivo empleado para el visionado:

\begin{tabular}{|lcc|}
\hline Sexo del espectador & + & Visualizaciones \\
Mujer & $63,0 \%$ \\
Hombre & $37,0 \%$ \\
\hline
\end{tabular}

Figura 3: Análisis cuantitativo de la serie. Sexo del usuario Fuente: Youtube Analitycs

\begin{tabular}{|c|c|c|c|}
\hline $\begin{array}{c}\text { Fuente de } \\
\text { tráfico }\end{array}$ & $\begin{array}{c}\text { Área } \\
\text { geográfica }\end{array}$ & $\begin{array}{c}\text { Edad del } \\
\text { espectador }\end{array}$ & $\begin{array}{c}\text { Sexo del } \\
\text { espectador }\end{array}$ \\
\hline Edad del espectador & & & Visualizaciones \\
\hline Entre 13 y 17 años & & & - \\
\hline Entre 18 y 24 años & & & $55,1 \%$ \\
\hline Entre 25 y 34 años & & & $16,9 \%$ \\
\hline Entre 35 y 44 años & & & $13,3 \%$ \\
\hline Entre 45 y 54 años & & & $11,4 \%$ \\
\hline Entre 55 y 64 años & & & $3,3 \%$ \\
\hline Más de 65 afios & & & - \\
\hline
\end{tabular}

Figura 4: Análisis cuantitativo de la serie. Edad del usuario Fuente: Youtube Analitycs 


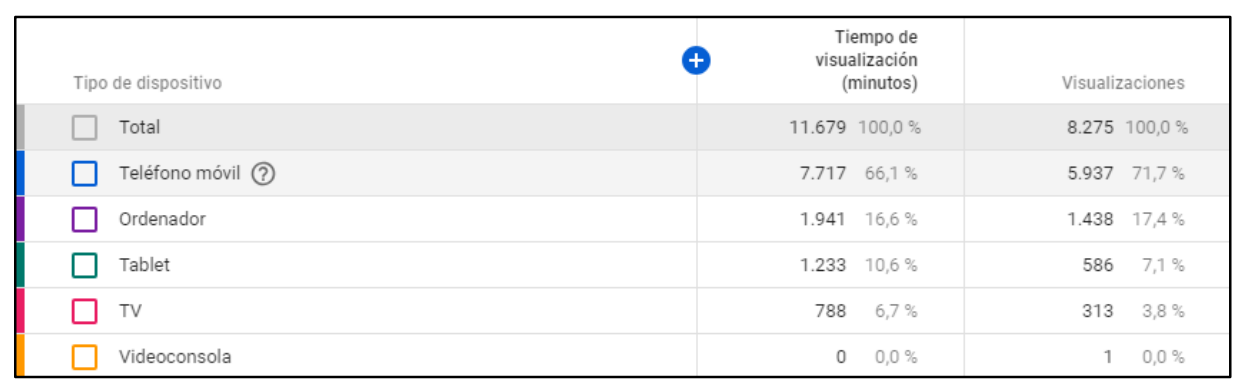

Figura 5: Análisis cuantitativo de la serie. Dispositivos

Fuente: Youtube Analitycs

La parte del entorno transmediático no tiene la posibilidad de análisis más allá de los likes, dislikes e interacciones, pero sí que es significativo que el capítulo final solo consiguió 185 espectadores. Esto indicó al alumnado que algo había fallado, aduciendo el bajo resultado a la dificultad narrativa de la narración y alto grado de compromiso que se pedía al usuario para poder llegar hasta el final.

\subsection{Reflexión profesor - alumnos}

Esta experiencia educativa no adquiere un sentido pleno hasta que no se analiza e investiga junto a sus protagonistas. Esta asignatura está situada al final de su formación universitaria, por lo tanto, a la hora de evaluar, hay que valorar si han sabido hacer uso de lo aprendido en otras muchas asignaturas (transversalidad), así como la relación de esta experiencia con su desarrollo competencial.

Dado que esta experiencia se lleva desarrollando desde el curso 2013-2014, a los 52 alumnos que han cursado la asignatura desde entonces se les planteó una encuesta de 23 preguntas acerca del desarrollo académico de la materia, que, en definitiva, supone una evaluación crítica de su asunción de competencias. Como todos los proyectos audiovisuales transmedia han salido adelante, el profesorado ha entendido que han cumplido con las competencias tanto las tres específicas como las diez generales, todas ellas detalladas anteriormente.

Las preguntas se respondían mediante escala Likert con valores entre 1 y 5 . La tasa de respuesta ha alcanzado el $86,5 \%$ (45 de los 52 estudiantes). En ninguna de las 23 preguntas la media ha bajado de 4 puntos sobre 5 . De aquí se desprende que el alumnado ha estado motivado y conforme con la labor docente. Cabe recordar que, junto con el examen teórico de la materia, ésta es la única actividad práctica a realizar, dado el notable volumen de trabajo desarrollado, tal y como se ha explicado en el epígrafe anterior. Los ítems evaluados con una mayor puntuación (ha variado entre los 4.5 y 4.8 puntos en los seis cursos analizados) han sido los siguientes: 
- "El profesor ha facilitado mi aprendizaje consiguiendo mejorar mis conocimientos y habilidades de forma."

- "El profesor ha fomentado mi participación e interés en las actividades planificadas de forma."

- "He comprendido los resultados de aprendizaje previstos en la asignatura de forma."

- "Mi grado de satisfacción con los resultados de aprendizaje que he alcanzado en la asignatura teniendo en cuenta los planificados en la misma es muy alto."

- "El profesor organiza y estructura bien las actividades que se realizan en clase (clases teóricas, seminarios, trabajo en grupo, presentación de trabajos, laboratorios, etc.) de forma."

El estudio ha querido ir más allá, por lo que el profesor responsable de la asignatura convocó un focus group con los 7 alumnos que han dado forma a la webserie Tiempo Muerto, una vez finalizado el proyecto y antes de conocer sus calificaciones finales, para evitar la influencia de la evaluación docente en sus respuestas. El focus se estructuró en tres ámbitos temáticos: planteamiento del proyecto, narrativa y producción, en primer lugar; resultados de visionado obtenidos, en segundo término; y resultados de aprendizaje individual y grupal basados en las competencias, en último término.

De este grupo de discusión emanaron puntos fuertes y débiles destacables. En primer lugar, que las competencias generales se trabajaron en diferente grado de desarrollo, en el sentido de que dos alumnas ejercieron mayor papel de liderazgo que algunos de sus compañeros. Ambas reseñaron que la experiencia les había permitido ejercer el rol de 'líder' o 'coordinador', algo novedoso durante su etapa universitaria, por cuanto en el resto de trabajos grupales la dinámica de funcionamiento consistía, simplemente, en repartirse las tareas. El resto de componentes aseveró que de forma tácita escogieron a estas dos personas como 'capitanes' por sus dotes de mando.

En lo concerniente al resto de competencias, los alumnos afirmaban haber trabajado y sentir que ese aprendizaje lo habían asumido e interiorizado, y reconocieron que gracias a este proyecto había comprobado de forma práctica y real algunos conocimientos asumidos durante años previos en otras asignaturas. Sintieron, de forma unánime, que el reto planteado por el profesorado les había permitido sentirse autónomos a la hora de decidir y crear. Concedieron que era necesaria la creación de unas pautas previas a modo de marco normativo, pero se sintieron con libertad de modificar cuestiones esenciales. Uno de los participantes indicó que el cuerpo docente había funcionado como un "jefe en la sombra", sin intervención directa, pero sabedor en todo momento de lo que iba aconteciendo. De este modo, confirmaron los resultados de la encuesta (realizada, 
además de a ellos, a compañeros de años anteriores), y no manifestaron ninguna propuesta de mejora.

Esto nos lleva directamente a poner en relación lo necesario de realizar actividades, que formen al alumno universitario de cara a su inserción en el mercado laboral, con el desarrollo de unos contenidos específicos que aborden la narratología transmedia para su futura puesta en práctica en sus futuros ámbitos de trabajo.

\section{CONCLUSIONES}

La experiencia ha resultado enriquecedora a tenor de los resultados metodológicos expuestos, tanto en lo referente a las encuestas y, especialmente, al focus group. Los alumnos han manifestado que el proyecto les ha permitido reordenar los conocimientos y competencias adquiridas en los cuatro años del Grado, haciendo uso de las diferentes herramientas y materiales. Estamos hablando, por lo tanto, de Entornos Personales de Aprendizaje y Entornos de Aprendizaje Abiertos.

No conviene olvidar tampoco que a medida que han pasado los cursos académicos, se han podido referenciar más ejemplos de narrativa transmedia en la televisión nacional, motivo por el que ha sido más fácil poder explicar al alumno en qué consiste este concepto, y, por ende, les ha sido más fácil desarrollar productos de este tipo y fijar la asunción de competencias.

Si bien es cierto que las visualizaciones han sido bajas en los últimos capítulos, tal y como vaticinaban los propios creadores por la idiosincrasia de su target, ninguno de ellos sintió que su trabajo quedase invalidado por este motivo, dado que fueron conscientes de sus limitaciones presupuestarias. Pero también es necesario trascender las estadísticas y fijar las conclusiones en el ámbito académico. La retroalimentación proporcionada por todo el alumnado de esta asignatura conduce a validar tanto la hipótesis principal como aquellas secundarias: el alumnado usa esta experiencia para poner en práctica todo lo aprendido durante los cuatro años de su etapa universitaria, de forma que esté mejor preparado para un entorno laboral que cada vez apuesta más por lo transmedia, y además lo hace todo solo usando su imaginación y los recursos técnicos de los que dispone en el aula.

De igual modo, el uso del Aprendizaje Significativo y la terminología alrededor de las dinámicas de Aula Invertida cobran sentido cuando se tratan proyectos de este calado. No vale solo con explicar mediante una lección magistral qué son las narrativas transmedia; además, hay que tener material al alcance del profesor para que éste lo pueda poner en circulación fuera de clase y los alumnos puedan llegar al aula con las ideas renovadas y los conceptos claros para poder ponerlos en práctica. En algunos casos, es simplemente subir vídeo o texto a la nube, en otros, poder establecer dinámicas fuera del aula con todos los integrantes del proceso comunicativo trabajando en línea para 
que quede registro del trabajo realizado y que los alumnos puedan volver a este contenido cuando sea necesario. Es necesario remarcar que esta premisa depende del grado de implicación de ambos agentes, por cuanto la primera semana de trabajo es crucial para testar la predisposición de los estudiantes.

Si este trabajo es bien realizado y dirigido por el docente, cuando el alumnado vuelva al aula, muchas de las ideas de producción ya estarán fijadas y tan solo habrá que poner énfasis en aquellos aspectos que estén menos claros o que generen dudas a la mayoría del grupo. Dudas que en todos los cursos académicos han surgido, por cuanto cada alumno responde a un perfil diferente al de sus compañeros. Pero estas diferencias en los perfiles son habituales en el ámbito profesional audiovisual.

De todas estas ideas emana la necesidad de proponer dinámicas y proyectos en las aulas que potencien las narrativas transmedia, ya que las posibilidades de relatos multiplataforma ayudan al alumno a la consecución de una diversidad competencial que va más allá de una única asignatura, sino que pone en práctica las labores del aprendizaje significativo. $Y$ es que el alumno de último curso se tiene que enfrentar no solo a un nuevo proceso creativo sino a poner en práctica todo lo aprendido en la carrera, en este caso, comunicación audiovisual, en sus diferentes ramas.

Se puede concluir estableciendo una relación de analogía entre la creación de redes de conocimientos y narrativas transmedia. Estas últimas se deben poder conectar con la audiencia a través de dispositivos móviles, evolucionan con el tiempo, son participativas, basadas en la nube e inclusivas. En cuestión de educación, se proponen cinco características del aprendizaje significativo y se catalogan como activas, constructivas, intencionales, auténticas y colaborativas. Misma esencia en distintos ámbitos.

En el aula no siempre hay una respuesta correcta unívoca, sobre todo cuando se trabaja con temas creativos. Por eso, debemos, como docentes, empezar a entender cuáles son los saberes que el alumno va a ser capaz de autoaprender y dirigir hacia la adquisición competencial con experiencias que fomenten su interés, al igual que en esta experiencia se invita a fomentar la participación del usuario.

\section{Referencias}

Ambrosino, M. A. (2017). Docencia y Narrativas Transmedia en la educación superior. Trayectorias Universitarias, 3(4), 12-19. Recuperado de http://bit.ly/2kmybGw

Avello Martínez, R. y Duart, J.M. (2016). Nuevas tendencias de aprendizaje colaborativo en elearning. Claves para su implementación efectiva. Estudios Pedagógicos XLII, N 1, 271282. Recuperado de http://bit.ly/2IPdQKe 
Ausín, V., Abella, V., Delgado, V., \& Hortigüela, D. (2016). Aprendizaje basado en proyectos a través de las TIC. Una experiencia de innovación docente desde las aulas universitarias. Formacion Universitaria, 9(3), 31-38. https://doi.org/10.4067/S071850062016000300005

Badia, A., \& García, C. (2006). Incorporación de las TIC en la enseñanza y el aprendizaje basados en la elaboración colaborativa de proyectos. Revista de Universidad y Sociedad Del Conocimiento, 3(2), 42-54. Recuperado de http://bit.ly/2kcV1A6

Belloch, C. (1998). Las Tecnologías de la Información y Comunicación en el Aprendizaje. Unidad de Tecnología Educativa (UTE)- Departamento de Métodos de Investigación y Diagnóstico En Educación., 1-9. https://doi.org/10.15446/rsap.v17n1.36935

Blank, W (1997). Authentic instruction. In W.Blank \& S. Harwells (Eds.), Promising practices for connecting high school to the real world. (p.15-21). Tampa, FL: University of South Florida

Cabero Almenara, J. (1998). Las Aportaciones De Las Nuevas Tecnologías a Las Instituciones De Formación Contínuas: Reflexiones Para Comenzar El Debate. Las Organizaciones Ante Los Retos Del Siglo XXI., (April), 1-7. Recuperado de http://bit.ly/2kgSjJV

Cabero Almenara, J. (2005). Cibersociedad y juventud: la cara oculta (buena) de la Luna. En Netbiblio (Eds.) Un nuevo sujeto para la sociedad de la información. Las Palmas de Gran Canaria: COMBYTE

Cabero Almenara, J. (2006). Pedagogical bases of e-learning. RUSC. Universities and Knowledge Society Journal, 3(1). https://doi.org/10.7238/rusc.v3i1.265

Calzadilla, M. E. (2002). Aprendizaje colaborativo y tecnologías de la información y la comunicación. Revista Iberoamericana de Educación, 10.

Chaves, A. L. (2001). Implicaciones educativas de la teoría sociocultural de Vigotsky. Revista Educación, 25(2), 59-65. Recuperado de http://bit.ly/2khBej4

Cebrián Herreros, M. (2009). Nuevas formas de comunicación: Cibermedios y medios móviles. Comunicar: Revista Científica Iberoamericana de Comunicación y Educación, 16(33), 10-13. https://doi.org/10.3916/c33-2009-01-001

Corchuelo Rodríguez, C. A. (2018). Gamificación en educación superior: experiencia innovadora para motivar estudiantes y dinamizar contenidos en el aula. Edutec. Revista Electrónica de Tecnología Educativa, (63), 29. https://doi.org/10.21556/edutec.2018.63.927

Cortizo Pérez, J. C., Carrero García, F., Monsalve Piqueras, B., Velasco Collado, A., Díaz del Dedo, L. I., \& Pérez Martín, J. (2011). Gamificación y Docencia: Lo que la Universidad tiene que aprender de los Videojuegos. VIII Jornadas Internacionales de Innovación Universitaria, 1-8.

Costa Sanchez, C., \& Piñeiro Otero, T. (2012). Nuevas narrativas audiovisuales: multiplataforma, crossmedia y transmedia. El caso de Águila Roja (RTVE). Revista ICONO14. Revista 
Científica de Comunicación y Tecnologías Emergentes, 10(2), 102. https://doi.org/10.7195/ri14.v10i2.156_

De la Fuente Prieto, J., Gómez, S. C., \& Borda, R. M. (2016). El inicio de la televisión transmedia en España: TVE y" Víctor Ros". Revista de La Asociación Española de Investigación de La Comunicación, 3(6), 28-42.

Duarte, A. (1998). Navegando a través de la información: diseño y evaluación de hipertextos para la enseñanza en contextos universitarios. Huelva: Facultad de Ciencias de la Educación, Tesis doctoral inédita.

Evans, E. (2011). Transmedia Television: Audiences, New Media, and Daily Life. New York: Routledge

Ferrés, J. (2012). La competencia mediática: propuesta articulada de dimensiones e indicadores. Comunicar: Revista Científica Iberoamericana de Comunicación y Educación, XIX(38), 75. https://doi.org/10.3916/C38-2012-02-08

Fernández, F., \& Fernández, M. (2016). Los docentes de la Generación Z y sus competencias digitales. Comunicar: Revista Científica Iberoamericana de Comunicación y Educación, (46), 97-105. https://doi.org/10.3916/C46-2016-10

Fidalgo Blanco, A., Sein-Echaluce Lacleta, M. L., \& García Peñalvo, F. J. (2017). Aprendizaje Basado en Retos en una asignatura académica universitaria. IE Comunicaciones: Revista Iberoamericana de Informática Educativa., 25(enero-junio), 1-8. Recuperado de http://bit.ly/2meVOkJ

Galvis, R. V. (2007). De un perfil docente tradicional a un perfil docente basado en competencias. Acción Pedagógica, 16(1), 48-57.

Guerrero-Pico, M., \& Scolari, C. A. (2016). Narrativas transmedia y contenidos generados por los usuarios: el caso de los crossovers. Cuadernos.Info, (38), 183-200. https://doi.org/10.7764/cdi.38.760

Gonzalez, A.P. (1998). Perspectivas de futuro en la utilización de las nuevas tecnologías en la formación ocupacional y de empresa. Pixel-Bit. Revista de Medios y Educación, 10, 736.

Grandío Perez, M. del M. (2015): "Indicadores para la evaluación de la alfabetización transmedia en los estudios universitarios de Comunicación", en Formación, perfil profesional y consumo de medios de alumnos en Comunicación, Fernando Peinado Miguel (Coord.). Cuadernos Artesanos de Comunicación, 77. La Laguna (Tenerife): Latina.

Gubern, R. (2000). El eros electrónico. Mexico: Taurus.

Gutiérrez Martín, A., \& Tyner, K. (2012). Educación para los medios, alfabetización mediática y competencia digital. Comunicar: Revista Científica Iberoamericana de Comunicación y Educación, XIX (38), 31-39. https://doi.org/10.5944/educ 
Harwell, S. (1997). Project-based learning, promising practices for connecting high school to the real world. Tampa, FL: University of South Florida.

Jenkins, H. (2009). The Revenge of the Origami Unicorn: Seven Principles of Transmedia Storytelling. Recuperado de http://bit.ly/2kJNLfi

Labrador, E., \& Villegas, E. (2016). Unir Gamificación y Experiencia de Usuario para mejorar la experiencia docente. RIED. Revista Iberoamericana de Educación a Distancia, 19(2), 125. https://doi.org/10.5944/ried.19.2.15748

Liuzzi, A. (2014). Transmedia Historytelling. De documentales Interactivos y Géneros Híbridos. En F. Irigaray y A. Lovato (Eds.) Hacia una Comunicación Transmedia. Rosario: UNR Editora

Maldonado Pérez, M. (2008). Aprendizaje basado en proyectos colaborativos. Una experiencia en educacion superior. Laurus, 14(28), 158-180. Recuperado de http://bit.ly/2kLcjom

Marc Guerrero. (2014). Webs televisivas y sus usuarios: un lugar para la narrativa transmedia. Los casos de "Águila Roja” y "Juego de Tronos" en España. Comunicación y Sociedad, (21). https://doi.org/10.1097/01.chi.0000138356.29099.f1

Marcelo-García, C., Yot-Domínguez, C., \& Mayor-Ruiz, C. (2015). University Teaching with Digital Technologies. Comunicar: Revista Científica Iberoamericana de Comunicación y Educación, 23(45), 117-124. https://doi.org/10.3916/c45-2015-12

Martí, J. A., Heydrich, M., Rojas, M., \& Hernández, A. (2010). Aprendizaje basado en proyectos: una experiencia de innovación docente. Universidad EAFIT, 46, 11-21.

Martínez García, M. del M., García Domingo, B., \& Quintanal Díaz, J. (2013). "El Perfil Del Profesor Universitario De Calidad Desde La Perspectiva Del Alumnado." Educación XX1, 9(0), 183-198. https://doi.org/10.5944/educxx1.9.0.325

Medina-Nicolalde, M. A., \& Tapia-Calvopiña, M. P. (2017). El aprendizaje basado en proyectos una oportunidad para trabajar interdisciplinariamente. OLIMPIA. Revista de La Facultad de Cultura Física de La Universidad de Granma, 14(46), 236-246.

Morales Bueno, P. (2018). Aprendizaje basado en problemas (ABP) y habilidades de pensamiento crítico ¿una relación vinculante? Revista Electrónica Interuniversitaria de Formación del Profesorado, 21(2), 91-108. DOI: http://dx.doi.org/10.6018/reifop.21.2.323371

Morales Vallejo, P. (2000). Medición de actitudes en psicología y educación: construcción de escalas y problemas metodológicos. Madrid: Universidad Pontificia Comillas.

Mittell, J. (2006). Narrative Complexity in Contemporary American Television. The Velvet Light Trap, 58(1), 29-40. https://doi.org/10.1353/vlt.2006.0032

Oliva, H. A. (2016). The gamification as a methodological strategy in the university educational context. Realidad y Reflexión, 44, 19. 
Ossorio Vega, M. Á. (2014). Aplicación de la narrativa transmedia en la enseñanza universitaria en España: Aprendizaje colaborativo, multiplataforma y multiformato. Revista Internacional de Tecnología, Ciencia y Sociedad, 3(2), 25-38. Recuperado de http://bit.ly/2kgSu83

Pérez, P. y Aldás, J. (dirs.) (2018). U-Ranking 2018. Synthetic Indicators of Spanish Universities. Valencia: IVIE y Fundación BBVA

Pérez-Mateo, M., Romero, M., \& Romeu-Fontanillas, T. (2014). La construcción colaborativa de proyectos como metodología para adquirir competencias digitales. Comunicar: Revista Científica Iberoamericana de Comunicación y Educación, 21(42), 15-23. https://doi.org/10.3916/C42-2014-01

Pérez-Rodríguez, M. A., \& Delgado-Ponce, Á. (2012). From digital and audiovisual competence to media competence: Dimensions and indicators. Comunicar: Revista Cientifica Iberoamericana de Comunicación y Educación, 20(39), 25-33. https://doi.org/10.3916/C39-2012-02-02

Porto Renó, D. (2011). Periodismo, redes sociales y transmediación. Razón y Palabra, 16(78).

Pratten, R. (2015). Getting Started with Transmedia Storytelling a practical guide for beginners 2nd edition. CreateSpace Independent Publishing Platform.

Rocha, L. M. (1998). Selected Self-Organization. Evolutionary Systems: Biological and Epistemological Perspectives on Selection and Self-Organization, 341-358.

Rodríguez Sánchez, M. (2011). Metodologías docentes en el EEES. De la clase magistral al portfolio. Tendencias Pedagógicas, (17), 83-103. Recuperado de http://bit.ly/2IQDr5s

Rodríguez-Ferrándiz, R., \& Tur-Viñes, V. (2015). Narraciones sin fronteras. Transmedia storytelling en la ficción, la información, el documental y el activismo social y político. Cuadernos Artesanos de Comunicación., 81, p.148.

Rodríguez-Mateos, D. y Hernández-Pérez, T. (2015). Televisión social en series de ficción y nuevos roles del documentalista audiovisual: el caso de "El Ministerio del Tiempo". Index comunicación 5(3), 95-120.

Rodríguez-Sandoval, E., Luna-Cortés, J., \& Mauricio Vargas-Solano, E. (2010). Evaluación de la estrategia del aprendizaje basado en proyectos. Educación y Educadores, 13(1), 13-25. https://doi.org/ISSN 0123-1294.

Romero-Calmache, M., \& Fanjul-Peyró, C. (2010). Advertising in the Digital Age: the Microsite as a Strategic Factor in On-line Advertising Campaigns. Comunicar: Revista Cientifica Iberoamericana de Comunicación y Educación, 17(34), 125-134. https://doi.org/10.3916/c34-2010-03-12

Scolari, C. A. (2009). Transmedia Storytelling: Implicit Consumers, Narrative Worlds, and Branding in Contemporary Media Production. International Journal of Communication, 3 , 586-606. 
Scolari, C. A. (2013) Narrativas Transmedia. Cuando todos los medios cuentan. Barcelona: Deusto.

Scolari, C. A. (2016). Informal Learning Strategies and Media Skills in the New Ecology of Communication. Telos. Cuadernos de Comunicación e Innovación, 1-9. Reuperado de http://bit.ly/2IPB17a

Scolari, C. A., \& Establés, M. J. (2017). El ministerio transmedia: Expansiones narrativas y culturas participativas. Palabra Clave, 20(4), 1008-1041. https://doi.org/10.5294/pacla.2017.20.4.7

Scolari, C. A. (2018) Transmedia literacy in the new media ecology: white paper. Barcelona: Universitat Pompeu Fabra

Siemens, G. 2004. A learning theory for the digital age. Recuperado de http://bit.ly/2kJGZ9t

Villalustre, L., \& Del Moral, M. E. (2015). Gamificación: Estrategia para optimizar el proceso de aprendizaje y la adquisición de competencias en contextos universitarios. Digital Education Review, (27), 13-31. Recuperado de http://bit.ly/2kA05Pv 


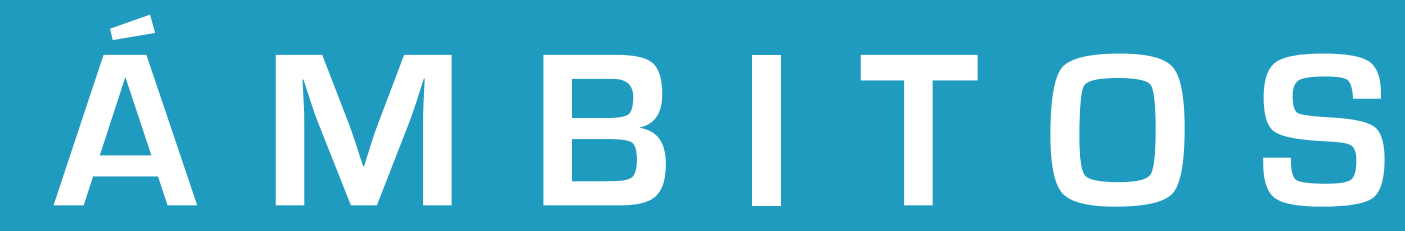

ISSN: 1139-1979 | ISSN digital: 1988-5733 | Depósito Legal: SE-1493-98

Revista Internacional de Comunicación editada por el Grupo de Investigación en Estructura, Historia y Contenidos de la Comunicación(GREHCCO) de la Universidad de Sevilla.

\section{ambitoscomunicacion@us.es}

http://institucional.us.es/ambitos

@RevistaAmbitos 